\title{
A SERVICE ORIENTED FRAMEWORK FOR MOBILE BUSINESS VIRTUAL COMMUNITIES
}

\author{
Julien Subercaze ${ }^{1}$, Pierre Maret ${ }^{1}$, \\ Jacques Calmet ${ }^{2}$, Pravin Pawar $^{3}$ \\ ${ }^{1}$ INSA-LYON, LIRIS (CNRS UMR 5205), FRANCE, julien.subercaze@liris.cnrs.fr, \\ pierre.maret@liris.cnrs.fr, \\ ${ }^{2}$ University of Karlsruhe (TH), GERMANY, calmet@ira.uka.de \\ ${ }^{3}$ University of Twente, THE NETHERLANDS, P. Pawar@utwente.nl
}

\begin{abstract}
The rise of the availability of a variety of mobile devices in the personal and professional domains leads to an increased need of middleware and organizational structures. Due to the recent improvement of the computing power of mobile devices, mobile users may now also act as service providers. We design a service-oriented architecture to introduce mobile virtual communities enabling mobile devices to be used also as service providers.
\end{abstract}

\section{INTRODUCTION}

The growing market of mobile devices, along with the ubiquitous availability of wireless and broadband access networks has opened a new era of market solutions. The sale of internet compatible devices is growing exponentially in recent years. For example, the sale of PDA units has reached the 17.7 million mark in $2006^{1}$, a $25.4 \%$ increase over 2005. Also, the manufacturer Research In Motion, that dominates the market of PDA devices with its Blackberry line of products, announced a $76.5 \%$ jump of sales in the first quarter of $2007^{2}$.

Simultaneously, the world of sensors is experiencing dramatic changes. For example, prices of imaging sensors since the introduction of CMOS sensors ${ }^{4}$ are falling sharply while their quality is increasing, and medical sensors prices also follow the same trend. As a Research and Marketers report noticed, "With the (actual) price of medical sensors (...), low cost and high volume sales have become the key to remaining competitive in the disposable sensors market" ${ }^{\prime 6}$.

\footnotetext{
${ }^{1} \mathrm{http}: / / \mathrm{www} . \mathrm{m}$-travel.com/news/2002/02/pda_sales_incre.html

${ }^{2}$ http://www.rim.net/investors/pdf/2007rim_ar.pdf

${ }^{3}$ http://www.computing.co.uk/computing/news/2193170/blackberry-sales-double

${ }^{4} \mathrm{http}: / / \mathrm{www}$. instat.com/abstract.asp?id=161\&SKU=IN0703690MI

${ }^{5}$ http://www.purchasing.com/article/CA6499634.htm

${ }^{6}$ http://www.researchandmarkets.com/reports/365353/world_market_for_sensor_opp ortunities_in.pdf
} 
Location sensors, i.e. GPS sensors, when not already embedded in mobile devices, are also available at much lower prices than only two years ago. A recent survey $^{7}$ shows a demand of mobile users to have an integrated GPS in their next mobile phone. Professional users are also fully concerned with these new tendencies. In the medical field, the use of specific external and internal nonintrusive sensors to treat patients, and the general use of mobile devices by healthcare providers are growing rapidly.

All of these changes show that we are witnessing the development of three trends in parallel: firstly, an increasing processing capability of mobile devices; secondly, an ever growing ubiquitous connectivity and thirdly, the widespread availability of sensors which can be interfaced to mobile devices respectively. These current trends assessed together result in the emergence of new user behaviors and open new opportunities for software products that will take full advantages of these features.

In this paper we describe our approach for mobile business virtual communities (MBVC). It follows the principles of service-oriented architecture that extends for business application our previous definition of virtual knowledge communities (Maret, 2008). Section 2 of the paper investigates related work on virtual organizations and communities, mobility and middleware support for web services. Section 3 describes the service-oriented features, while section 4 describes the mobile virtual communities platform architecture, this extends an off the shelf open source community software to include our service oriented architecture. In section 5 we present an example of MBVC based on this architecture. Finally we present some concluding comments in section 6 .

\section{RELATED WORKS}

Current investigations on virtual communities and virtual organizations focus often on the service oriented architecture. (Baglietto 2005) presents such an architecture implemented in the case of cargo and transport business area.(Shan 2006) defines a high level visual language for the description of service compositions in virtual organisations for non expert users. None of these architectures take the mobility feature into account.

Web services can be embedded on mobile devices, using mobile middleware such as those proposed in (Pratistha, 2003), (Srirama, 2006) or (Pawar, 2007). In our work, we rely on the middleware developed by Pawar (2007), which was initialy implemented for the remote patient telemonitoring in the mobile health domain.

Virtual enterprises have been defined as "a temporary consortium of autonomous, diverse and possibly geographically dispersed organizations that pool their resources to meet short-term objectives and exploit fast-changing market trends" (Davulcu, 1999).

Creation of virtual enterprises has to be made through a contract. Contracts could take into account business and/or legal issues. (Metso, 2005) proposes a B2B web service based middleware to set up business level contracts between the different

\footnotetext{
${ }^{7}$ http://www.itnews.com.au/News/67338,gps-to-boost-mobile-phone-sales.aspx
} 
actors. (van den Heuvel, 2003) proposes an architecture that coordinates webservice based organizations with contracts, focusing on business and legal issues.

Mobile virtual communities are introduced in (El Morr, 2007) and (Kawash, 2007). However, interfacing mobile virtual communities with service oriented architectures for virtual enterprises has not been fully explored. The aim of this paper is to combine virtual communities' facilities and middleware support for mobile web services. We use also contracts and negotiations tools for SOA, which lead then to the creation of MBVC.

\section{SERVICE ORIENTED ARCHITECTURE}

In service oriented architecture (SOA), the different entities taking part in the system (also called actors) are either 'service providers' or 'service consumers'. This is not exclusive: actors can also be both providers and consumers.. A service is a unit of work which can be done by a service provider to achieve desired end results, for a service consumer. In SOA, mobile devices usually play the role of service consumers. Due to the evolution of the computing power, connectivity ability and storage capacity, the role of mobile devices has to be reconsidered. Indeed, we use mobile devices also as service providers. We will now first present the architecture for SOA mobile business virtual communities and then analyze the creation process and the use of a virtual community within the SOA.

A service federation approach has been identified in (Camarinha-Matos, 2005).The partners are service providers, they form a community of providers and the resulting enterprise is a complex composition and orchestration of their services.

We propose a so-called Community Platform, based on the principles of SOA and using open source community software. Two types of actors are fundamental to this platform. The first ones are actors providing services and willing to propose them for the creation of Mobile Business Virtual Communities (MBVCs). They form the community of Providers. The providers could be fixed as well as mobile. The second type of actors is composed of the end-users (or service consumers), using MBVCs, i.e. using some of the proposed services. End-users form the second community.

Combining both communities (Providers and End-users) into one platform leads to some valuable advantages: The community platform considers the user profiles and actions, as well as the user's rights, and then selects the most appropriate service for this user. This is clearly of interest for business applications, but it can also be implemented towards medical assistance for instance (Pawar, 2008). Since our architecture includes also mobile devices as service providers, end-users can invoke these mobile services (Fig.1). 


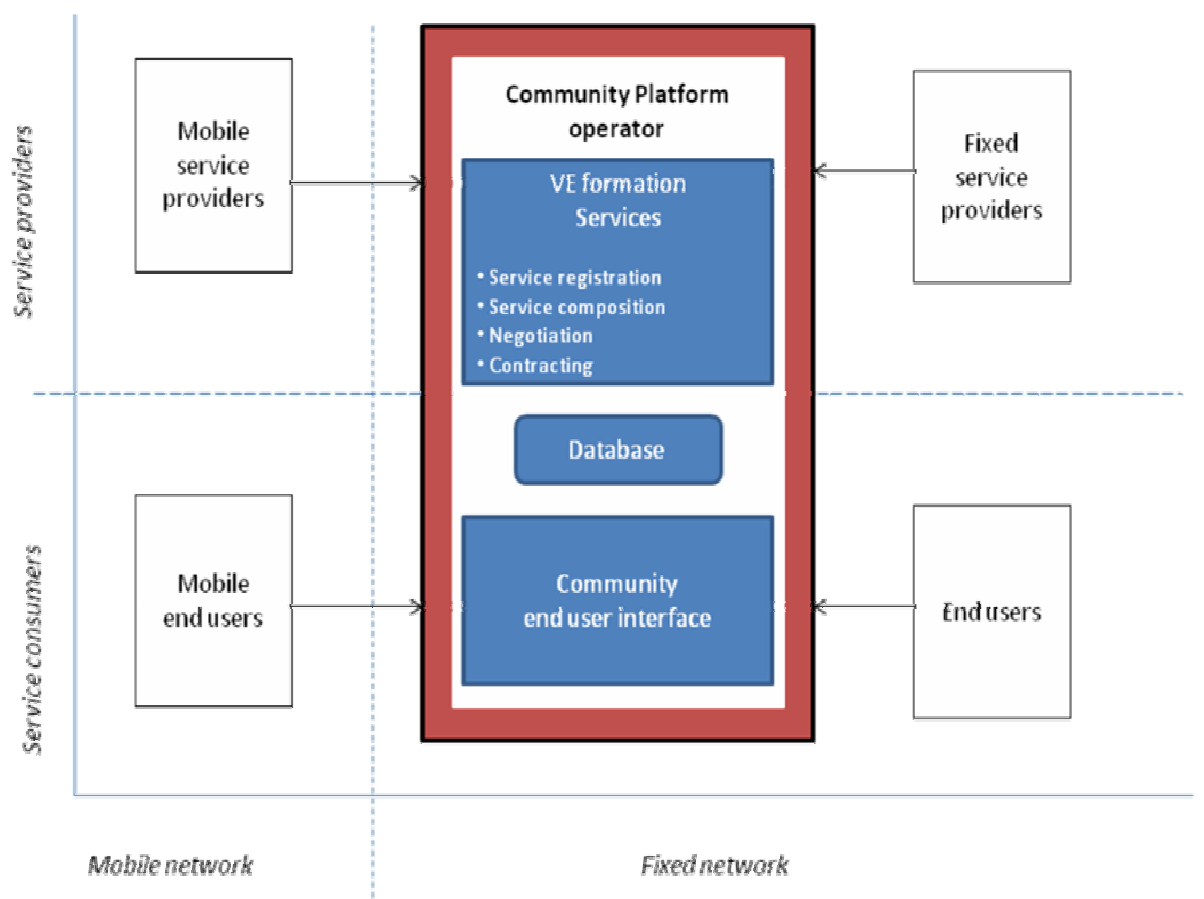

Figure 1: Fixed and mobile service providers and consumers around the community platform.

\section{MOBILE BUSINESS VIRTUAL COMMUNITIES}

\subsection{Creation of Mobile Business Virtual Communities}

The creation of the MBVC (fig. 2) is organized by the community platform operator (CPO); the first step is the registration of the services on the platform. Any user could register as a service provider. The next step consists of the partners' aggregation. This is controlled by one of the partner, using profile information, history and all other data it can find. Partners finalize their engagements in the contracting step. The Virtual Enterprises (VE) is then published. 


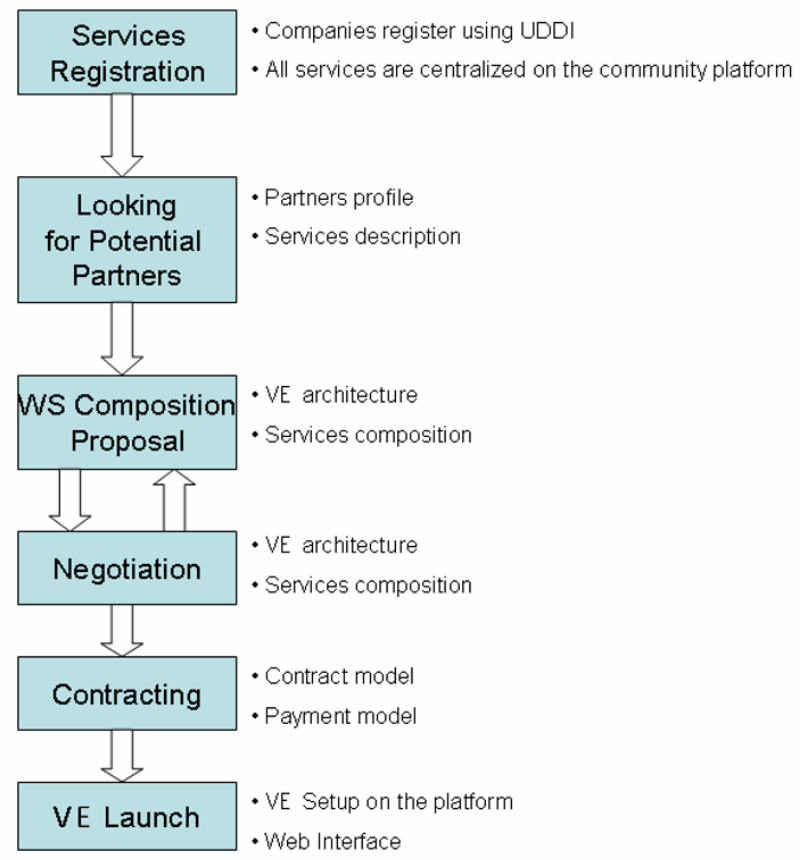

Figure2 : VE Creation Diagram

The community platform operator (CPO) provides the facilities to the service providers to create virtual enterprise within the platform. Revenue sources of the CPO are decided during the contract step. Revenues of the CPO may be for example calculated on a percentage on transactions, or on the duration of the community or on any agreed upon procedure.

The list of VE is presented on the community platform. End-users decide then to act as members of a given VE. Members can use fixed or mobile devices and they can participate using fixed or mobile services in sharing data and documents within the communities. On the platform, several communities are identified. The service providers form the community of providers. On the other side, end users are also forming a community. Then around the VE formed by the service providers, several communities (MBVC) involving the end users are made up. We demonstrate those communities in the next section.

\subsection{Implementation}

Our implementation is based upon an off-the-shelf open source community platform. The platform Dolphin ${ }^{8}$ was tuned to fulfill the requirements of the Service Oriented Architecture. We select to use Web Services technologies for this implementation and we rely on the PHP-based library NuSoap ${ }^{9}$ to interface Dolphin

\footnotetext{
${ }^{8}$ http://www.boonex.com/products/dolphin/

${ }^{9}$ http://sourceforge.net/projects/nusoap/
} 
native PHP with Web Services. The VE formation is not yet fully implemented, so it remains controlled by the CPO. VEs are created and they include mobile services. Users can become members of these communities; they can participate and make use of the services.

\subsection{Problems related to mobile devices}

The use of mobile devices involves numerous problems regarding connectivity and quality of service. Problems of connectivity are solved by using the Mobile Service Platform (MSP) middleware (Pawar 2007). MSP exploit multi-homing support for the resource constrained and handheld mobile devices and integrates a QoS context source to take QoS issues into account.

\section{USE CASES}

\subsection{Content server within communities}

User generated content is one of the growing business in the web economy. This generates a considerable amount of traffic, composed of the content generated by user. This content production is usually not rewarded. They provide storage and sharing facilities and the contents are generated for free by users, although those websites generate subsequent revenue through displayed advertisements. Using our platform, providers of pictures or videos register as content providers. The proposed service is in this case the access to pictures or videos. Members of this MBVC can send requests to the mobile service in order to get some pictures or videos. Members could be both content consumers and content producers. The community platform guarantees that content producers are rewarded according to the traffic generated by their provided content. End-users are thus encouraged to provide original contents.

The design of this MBVC encompassing mobile services matches our platform architecture. It consists of 1) the basic Virtual Communities features 2) the content provider service and 3 ) the billing service (fig. 3). Notice that the billing service is part of the platform because it is shared by all MBVCs. Others services can be added to the communities, such as the file storage service, the video encoding service and context advertisement service. Providers can be companies specializing in either fixed or mobile services or in both of them.

This business case enabled by our architecture is a win-win situation. Users earn money from the content they provide and the file storage and video encoding companies. High quality contents are also encouraged and this is also valuable for all: end-user, content providers, service providers and platform operator. 


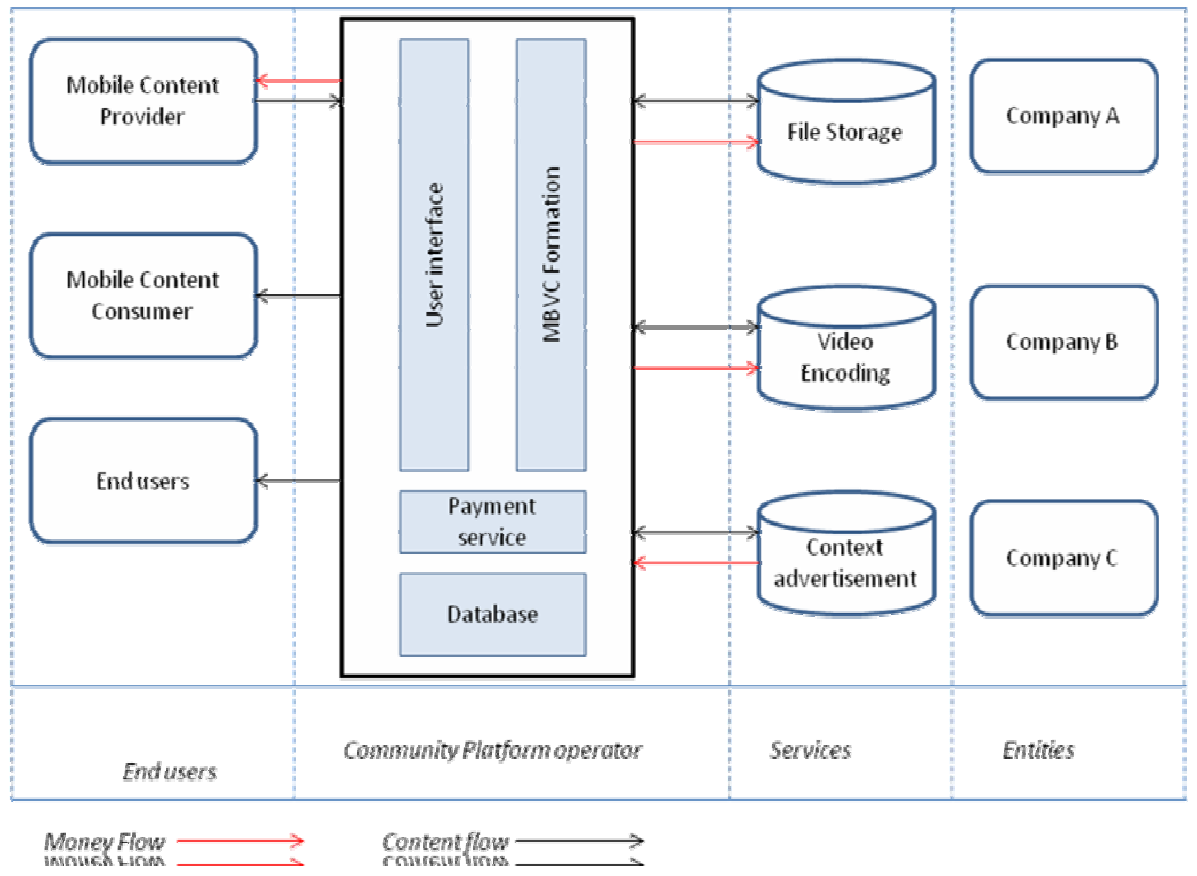

Figure 3: Use-case "Video sharing"

\subsection{Decision making support within communities}

Employees of a company form a community centered on its activity. Knowledge management into companies is a strategic issue, especially when decisions must be taken with limited time and incomplete knowledge.

Models and implementations of decision making processes have been proposed in several trivial or complex forms. (Yang 2007) gives an example of a service that makes decision with uncertain knowledge based on Bayesian networks.

Let us imagine a company's employee loading a decision making process as a service on her/his mobile device. Once this device is recognized within the company's community on the community platform, the service (decision making process) can be declared and then be used as a mobile service.

\section{CONCLUSION}

In this paper we introduce mobile virtual business communities based on a serviceoriented architecture. We design an architecture centered upon the community platform operator. This platform supports the creation of virtual communities based on a service federation approach. Relying on an adequate middleware we integrate mobile devices as services providers and include them as active resources shared within virtual communities. We outline the architecture and our first use case considers mobile service provider in a service federation approach organization. 
Future works will concentrate on the creation process of communities providing composed services and interfaces to create easily web based business community using mashups ${ }^{10}$ editors.

\section{REFERENCES}

P. Maret, J. Subercaze, and J. Calmet, Peer-to-Peer Model for Virtual Knowledge Communities, Proc. of AIKED 2008, Cambridge, UK, 365-370, 2008.

C. El Morr, J. Kawash. Mobile virtual communities research: a synthesis of current trends and a look at future perspectives. IJWBC, 3, 386-403, 2007.

Jalal Kawash, C. El Morr . A novel collaboration model for mobile virtual communities. IJWBC , 427-447, 2007.Pierpaolo Baglietto, Massimo Maresca, Andrea Parodi, and Nicola Zingirian.

Stepwise deployment methodology of a service oriented architecture for business communities. Information \& Software Technology, 47(6):427-436, 2005.

César Garita, Ulises Agiiero, Lorenzo Guadamuz, Network-Centric Collaboration and Supporting Frameworks, chapter Areito: A Development Platform For Virtual Learning Communities, pages 323 - 332. 2006.

Janne Metso and Lea Kutvonen. Managing virtual organizations with contracts. In Workshop on Contract Architectures and Languages (CoALa 2005), 2005.

Pravin Pawar, Bert-Jan van Beijnum, Arjan J. H. Peddemors, and Aart van Halteren. Contextaware middleware support for the nomadic mobile services on multi-homed handheld mobile devices. In ISCC, pages 341-348. IEEE, 2007.

I. Made Putera Pratistha, Nicholas Nicoloudis, and Simon Cuce. A microservices framework on mobile devices. In Liang-Jie Zhang, editor, IWCS, pages 320-325. CSREA Press, 2003

Baohua Shan, Yanbo Han, and Hongcui Wang. Enabling virtual organizations with an agentmediated service framework. In CSCWD, pages 626-631. IEEE, 2006.

Satish Narayana Srirama, Matthias Jarke, and Wolfgang Prinz. Mobile web service provisioning. In AICT/ICIW, page 120. IEEE Computer Society, 2006.

Willem-Jan van den Heuvel and Hans Weigand. Coordinating web-service enabled business transactions with contracts. In Johann Eder and Michele Missikov, editors, CAiSE, volume 2681 of Lecture Notes in Computer Science, pages 568-583. Springer, 2003.

Lai Xu and Paul de Vrieze. Fundaments of virtual organization E-contracting. In Luis M. Camarinha-Matos, Hamideh Afsarmanesh, Paulo Novais, and Cesar Analide, editors, Virtual Enterprises and Collaborative Networks, volume 243 of IFIP, pages 209-216. Springer, 2007.

Yang Yi, A Framework for Decision Support Systems Adapted to Uncertain Knowledge, PhD Dissertation Karlsruhe University, 2007.

Hasan Davulcu, Michael Kifer, L. Robert Pokorny, C. R. Ramakrishnan, I. V. Ramakrishnan, and Steven Dawson. Modeling and analysis of interactions in virtual enterprises. In RIDE, pages 12-18, 1999

${ }^{10}$ http://en.wikipedia.org/wiki/Mashup_\%28web_application_hybrid\%29 